\title{
Large amplitude oscillatory measurements as mechanical characterization methods for soft elastomers
}

Skov, Anne Ladegaard

Published in:

Proceedings of SPIE - The International Society for Optical Engineering

Link to article, DOI:

$10.1117 / 12.912335$

Publication date:

2012

Document Version

Early version, also known as pre-print

Link back to DTU Orbit

Citation (APA):

Skov, A. L. (2012). Large amplitude oscillatory measurements as mechanical characterization methods for soft elastomers. Proceedings of SPIE - The International Society for Optical Engineering, 8340, 83401C. https://doi.org/10.1117/12.912335

\section{General rights}

Copyright and moral rights for the publications made accessible in the public portal are retained by the authors and/or other copyright owners and it is a condition of accessing publications that users recognise and abide by the legal requirements associated with these rights.

- Users may download and print one copy of any publication from the public portal for the purpose of private study or research.

- You may not further distribute the material or use it for any profit-making activity or commercial gain

- You may freely distribute the URL identifying the publication in the public portal 
Return to your MySPIE To Do List at http://myspie.org and approve or disapprove this submission. Your manuscript will not be published without this approval. Please contact author_help@spie.org with any questions or concerns.

\title{
Large amplitude oscillatory measurements as mechanical characterization methods for soft elastomers
}

\author{
Anne Ladegaard Skov \\ Department of Chemical and Biochemical Engineering, DTU
}

\begin{abstract}
Mechanical characterization of soft elastomers is usually done either by traditional shear rheometry in the linear viscoelastic (LVE) regime (i.e. low strains) or by extensional rheology in the nonlinear regime. However, in many commercially available rheometers for nonlinear extensions the measurements rely on certain assumptions such as a predefined shape alteration and are very hard to perform on soft elastomers in most cases. The LVE data provides information on important parameters for DEAP purposes such as the Young's modulus and the tendency to viscous dissipation (at low strains only) but provides no information on the strain hardening or softening effects at larger strains, and the mechanical breakdown strength. Therefore it is obvious that LVE can not be used as the single mechanical characterization tool in large strain applications. We show how the data set of LVE, and large amplitude oscillating elongation (LAOE) ${ }^{1}$ and planar elongation ${ }^{2,3}$ make the ideal set of experiments to evaluate the mechanical performance of DEAPs. We evaluate the mechanical performance of several soft elastomers applicable for DEAP purposes such as poly(propyleneoxide) (PPO) networks ${ }^{3,4}$ and traditional unfilled silicone (PDMS) networks ${ }^{5}$.
\end{abstract}

Keywords: Elastomers, elasticity, loss modulus, storage modulus, material functions, mechanical breakdown.

\section{INTRODUCTION}

Elastomers are polymeric networks and are well known in many applications ranging from hard and brittle rubbers to soft and fragile gels. The softness of a network is a result of - amongst other- the applied polymer but also of the applied stoichiometry of the reactants since relatively large amounts of network imperfections can be introduced by unstoichiometric conditions. By network imperfections, we mean all the network chains which are not elastically active, i.e. connected to the infinite network in both ends, and hence does not contribute to the elastic modulus at low frequencies. The network imperfections will cause time-dependent properties of the networks as well as cause viscous dissipation within the material. Therefore it is very important to carefully evaluate the rheological behavior of a material to evaluate the applicability for use as DEAP material.

Rheological characterization is traditionally carried out using simple controlled methods such as steady shear, stress relaxation, creep, oscillatory shear and steady extension. The data from these tests are subsequently quantified by use of material functions such as the steady viscosity $(\eta(t))$, the creep compliance $(J(t))$, the relaxation modulus $(G(t))$, the storage $\left(G^{\prime}(\omega)\right)$ and the loss modulus $\left(G^{\prime \prime}(\omega)\right)$, respectively, where $t$ is the time and $\omega$ is the angular frequency of the imposed oscillation. For specific applications more advanced tests can be designed, e.g. to describe the effect of prestrain in elastomers. Or alternatively for the determination of the elastic modulus $\left(G^{\prime} G^{\prime}(\omega \rightarrow 0)\right)$ of thin films as the elastic modulus will not always be identical to that of bulk material as surface effects come into play and traditional rheological methods can not be directly applied. For this purpose a newly developed technique, the so-called buckling based metrology has been developed by Wilder et $\mathrm{al}^{6}$. It will, however, not be treated here where we focus on methods to determine bulk properties.

Soft polymer networks can possess both almost pure elastic as well as viscoelastic character, where the dominating behaviour depends on the applied time scale. Networks, which consist of both permanent crosslinks and transient entanglements originating from the substructures, will behave more like a polymer melt at short time scales, but on long time scales, it behaves as a rubber. Typical material stress-strain behaviours are illustrated in Figure 1 where the straight 
Return to your MySPIE To Do List at http://myspie.org and approve or disapprove this submission. Your manuscript will not be published without this approval. Please contact author_help@spie.org with any questions or concerns.

line indicates a linear dependency between the stress and the applied strain which indicates no hysteresis and hence characterizes a completely elastic material.

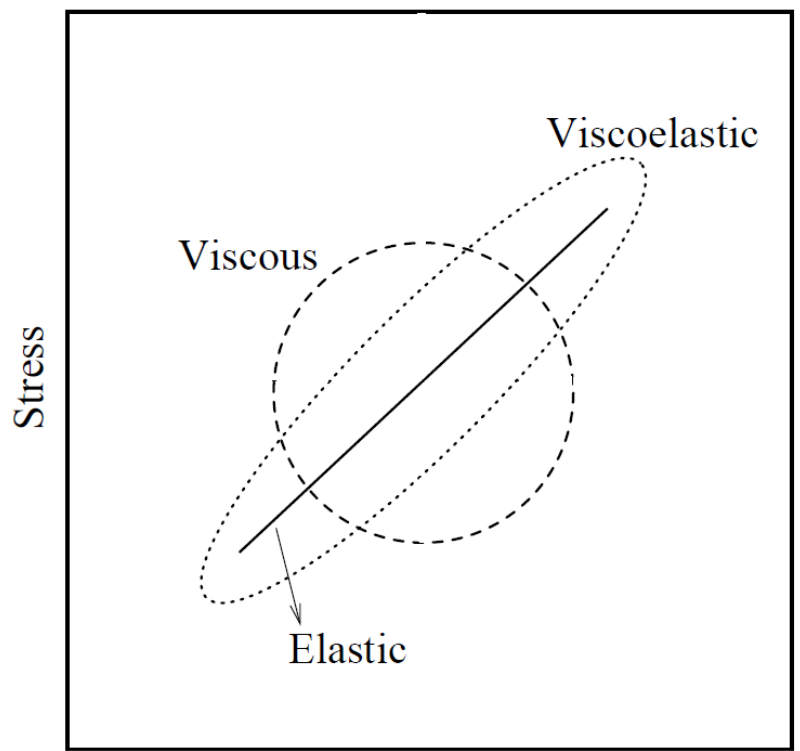

Strain

Figure. 1 The stress-strain behavior during steady-state cycles of oscillation for different categories of materials, namely purely elastic and purely viscous with the viscoelastic material combining the features of the two before-mentioned materials. Most soft elastomers are highly viscoelastic

Mechanical characterization of soft elastomers is usually done either by traditional shear rheometry in the linear viscoelastic (LVE) regime (i.e. low strains) or by extensional rheology in the nonlinear regime, where an example is the Sentmanat add-on device to traditional shear rheometers, which is illustrated in figure 2.

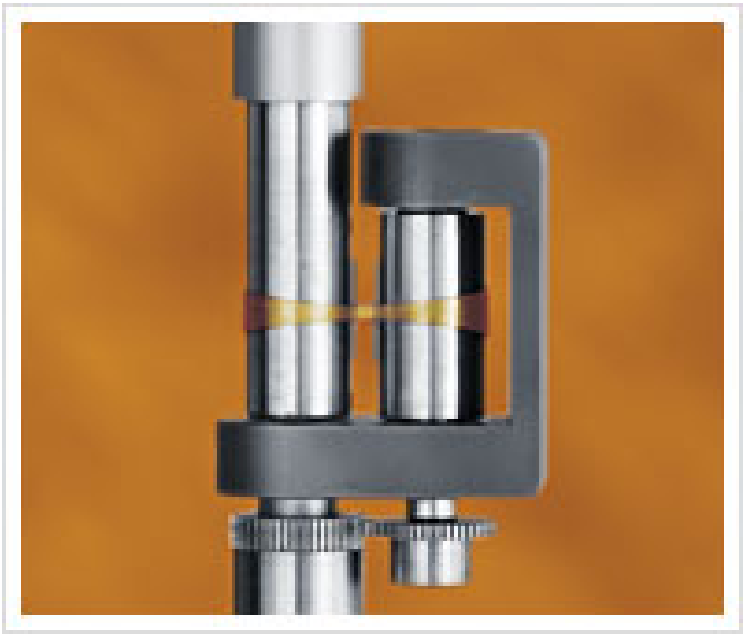

Figure 2: The Sentmanat add-on device to traditional shear rheometers from Xpansion Instruments. 
Return to your MySPIE To Do List at http://myspie.org and approve or disapprove this submission. Your manuscript will not be published without this approval. Please contact author_help@spie.org with any questions or concerns.

However, in many commercially available rheometers for nonlinear extensions the measurements rely on certain assumptions such as a predefined shape alteration and are very hard to perform on soft elastomers in most cases. The Sentmanat rheometer can for example not be used for reversing flows for soft, sticky elastomers since the elastomers will stick to the drums in reverse mode and buckle in an irreproducible way.

The LVE data provides information on important parameters for DEAP purposes such as the Young's modulus ( $\mathrm{Y}=3 \mathrm{G}$ in the case of incompressible materials) and the tendency to viscous dissipation (at low strains only) but provides no information on the strain hardening or softening effects at larger strains, and the mechanical breakdown strength. Therefore it is obvious that LVE can not be used as the single mechanical characterization tool for large strain applications. We show in the following that the data set of LVE, and large amplitude oscillating elongation (LAOE) ${ }^{1}$ and planar elongation ${ }^{2,3}$ make the ideal set of experiments to evaluate the mechanical performance of DEAPs. The oscillation modes furthermore allow us directly to calculate the dissipated mechanical energy within the material.

The tear strength of the materials can also be measured for both large amplitude methods by increasing the strain at constant strain rate. It is, however, important to emphasize that the tear strength should not necessarily be identical for the two methods as the flow is much stronger for the planar elongation method than for the uniaxial method. Furthermore the frequency dependency of the tear strength can be evaluated. The tests are, however, destructive so such experiments will be very time-consuming and traditional methods may suffice for the tear strength determination. If the DEAP is applied in a setting where one dimension is strictly fixed, it may be necessary to evaluate the tear strength in the planar elongation mode since smaller breakdown less strength can be expected.

\section{DESCRIPTION OF THE THREE METHODS}

In the following subsections we will summarize the main features of three experimental methods which in combination are capable of predicting the nonlinear mechanical behaviour of elastomers applied for e.g. DEAPs in different operation conditions.

\subsection{Linear Viscoelastic (LVE) measurements}

Linear viscoelastic measurements are performed by applying sinusoidal strain with frequency $\omega$ and strain amplitude $\gamma_{0}$ to the sample:

$$
\gamma=\gamma_{0} \sin \omega t
$$

The linear response of material in terms of stress can be written as:

$$
\sigma=\sigma_{0} \sin (\omega t+\delta)
$$

where $\delta$ is the phase lag. The response given by the above equation is usually observed at low amplitudes of strain, which for silicone elastomers usually have an upper limit of approximately 5-10\%. At larger strain amplitudes, nonlinear effects will interfere. Based on the strain imposed and the resulting stress measured, material functions can be used to quantify the material behavior. Two important material functions are the storage modulus, which is based on the amplitude of in-phase stress (hence a direct measure for the elasticity) and the loss modulus, which is based on the outof-phase stress (hence a direct measure for the viscous (loss) behavior). Based on these material functions, eq 2 can be written as:

$$
\sigma=\mathrm{G}^{\prime}(\omega) \sin \omega t+\mathrm{G}^{\prime \prime}(\omega) \cos \omega t
$$

LVE diagrams are measurements of G' and G" as function of frequency and they reveal little information for traditional hard elastomers since the storage modulus is usually constant over investigated frequency regime for commercially available shear rheometers (usually 0.001 to $100 \mathrm{~Hz}$ ) and the loss modulus is several decades lower than the storage modulus. However, measurements on soft elastomers reveal crucial information on the materials as the materials possess time dependent properties (determined by the time constant within the materials). An example of an LVE diagram for a soft poly(propyleneoxide) (PPO) network can be seen in figure 3 . 
Return to your MySPIE To Do List at http://myspie.org and approve or disapprove this submission. Your manuscript will not be published without this approval. Please contact author_help@spie.org with any questions or concerns.

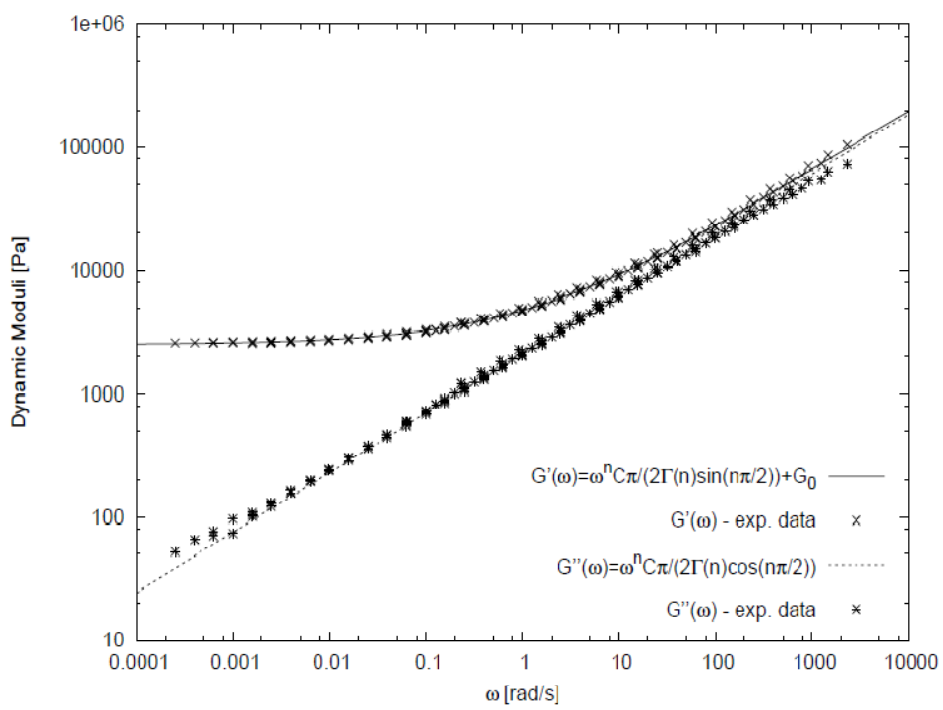

Figure 3. LVE of a poly(propyleneoxide) (PPO) network at $25^{\circ} \mathrm{C}$. The parameters $G_{0}, C$ and $n$ are determined with a least square fit of the expression for $G^{\prime}(\omega)$ to the measured data. $G_{0}=2500 \pm 60 \mathrm{~Pa}, C=1800 \pm 60 \mathrm{~Pa} \mathrm{~s}{ }^{n}$ and $n=0.484 \pm 0.007$. The stoichiometric ratio, $r$ equals $0.57 .^{4}$

Simple and more advanced models can be used to interpret these data such as the Ogden model $^{7}$, the gel equation ${ }^{2,8}$, the phonon fluctuation model ${ }^{9,10}$, the double tube model $^{11}$ and several others. In the modified gel equation proposed by the work of Jensen et $\mathrm{al}^{4}$ the storage modulus can be described as:

$$
\mathrm{G}^{\prime}(\omega)=C \omega^{n} \pi /(2 \Gamma(n) \sin (n \pi / 2))+\mathrm{G}_{0}
$$

where $\mathrm{C}$ is a measure for the stiffness of the network and $n$ is a relaxation exponent which are parameters from the original gel equation (Winter). $\mathrm{G}_{0}$ is the shear modulus.

The parameters $\mathrm{C}, \mathrm{n}$ and $\mathrm{G}_{0}$ are fitted simultaneously from the LVE spectrum. After the determination of the parameters the model can be used for predicting the low strain limit behavior as well as a characteristic time $\tau$ can be determined from the frequency at which there is an upturn in G'. For the very soft PPO elastomer investigated in the work of Jensen et al. ${ }^{4}$ the data can be summarized as seen in table 1 .

Table 1: Details of the PPO network sample extracted from the LVE diagram shown in figure 3. $\mathrm{r}$ is the stoichiometric imbalance for the soft elastomer. The other parameters are determined directly from the modified gel equation (eq 4).

\begin{tabular}{c|c} 
& Sample A \\
\hline $\mathrm{r}$ & 0.57 \\
$\mathrm{G}_{0}[\mathrm{~Pa}]$ & $2500 \pm 60$ \\
$\mathrm{C}\left[\mathrm{Pa} \cdot \mathrm{s}^{\mathrm{n}}\right]$ & $1800 \pm 60$ \\
$n$ & $0.484 \pm 0.007$ \\
$\tau=\left(C / G_{0}\right)^{1 / n}[s]$ & $0.5072 \pm 0.0007$
\end{tabular}

These data can then be used in combination with nonlinear models to interpret the nonlinear behavior if this is not directly determined by nonlinear measurements as presented in the next two experimental methods. Finite element method (FEM) modeling was also applied in the work of Jensen et al. ${ }^{4}$

For an extensive list of available LVE measurements on different silicone elastomers, see Larsen et al. ${ }^{12}$ 
Return to your MySPIE To Do List at http://myspie.org and approve or disapprove this submission. Your manuscript will not be published without this approval. Please contact author_help@spie.org with any questions or concerns.

\subsection{Oscillating Planar Elongational Measurements}

Planar elongation is in general given by the flow field $\lambda_{x}=\lambda, \lambda_{y}=1$ and $\lambda_{z}=1 / \lambda$, where $\lambda$ is the imposed relative extension and the index $i$ refers to the direction in an orthogonal coordinate system. The value of the extension in the z-direction comes from the assumption of incompressibility $\lambda_{x} \lambda_{y} \lambda_{z}=1$.

Planar elongation is often measured by methods where the sample is clamped in two dimensions, and then one of the fixed directions is allowed to move ${ }^{13,14}$. Clamping is more or less impossible for soft elastomers. Therefore Jensen et al ${ }^{4}$ developed a new technique not relying on clamps to measure planar elongation based on the original idea of Laun and Schuch for polymer melts ${ }^{15}$. The principle is to extend a circular annulus of material, which in the case of the elastomer is formed by a strip of the material around the device (figure 4) where the circumference is kept constant by increasing the pressure inside the annulus. This is, however, not necessary for elastomers where ambient pressure inside the device is sufficient to keep the elastomer vertically straight. The ambient pressure is ensured by holes in the device at both the top and bottom, see figure 4.

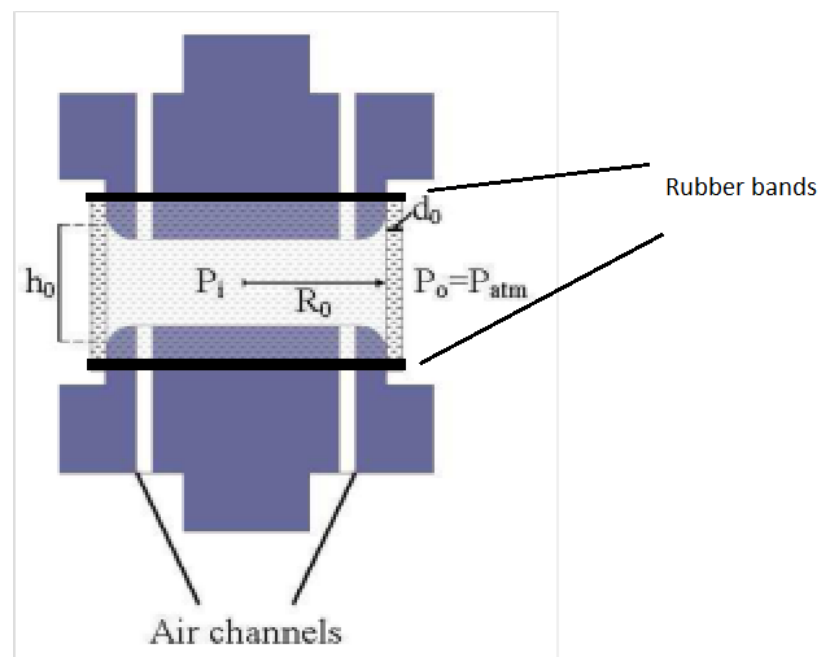

Figure 4. Side-view of the planar elongational fixture (PEF). The elastomer film strip has a slightly larger length than the circumference of the device. The PPO elastomer is very sticky and self-adhering as well as it adheres to steel. For non-sticky materials rubber bands are needed to fix the soft elastomer at the top and at the bottom plate.

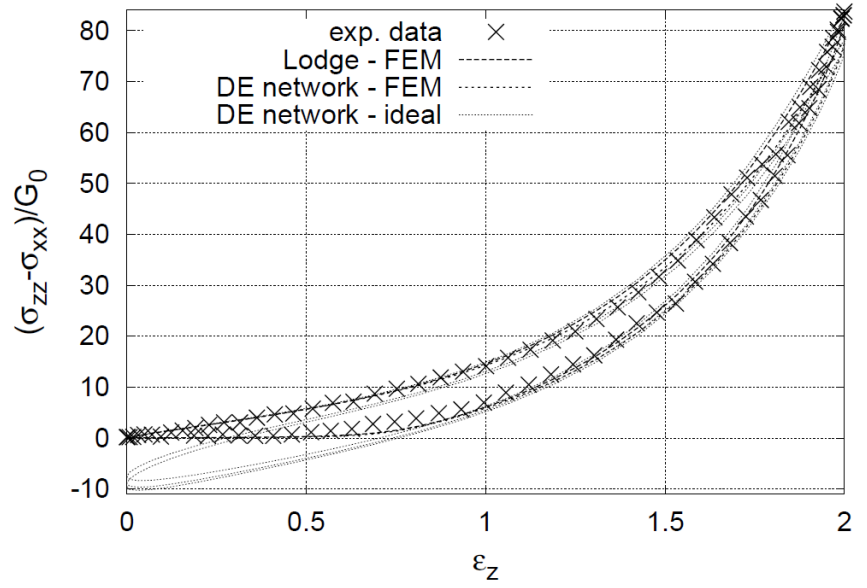

Figure 5. Measured stress differences obtained from the PPO elastomer for a nonlinear oscillating strain with a frequency of $\Omega=0.4$. $\pi \mathrm{rad} / \mathrm{s}$. The Hencky strain $\varepsilon$ is the logarithmic strain such that $\varepsilon=2$ means a relative extension in the $\mathrm{z}$-direction of $\lambda=\exp (2)=7.4$. The lines are the numerical FEM results, obtained with different nonlinear models. For more details see Jensen et $\mathrm{al}^{4}$ 
Return to your MySPIE To Do List at http://myspie.org and approve or disapprove this submission. Your manuscript will not be published without this approval. Please contact author_help@spie.org with any questions or concerns.

\subsection{Large amplitude oscillatory elongational (LAOE) experiments}

Uniaxial elongation is in general given by the flow field of $\lambda_{z}=\lambda$, and $\lambda_{x}=\lambda_{y}=1 / \lambda^{1 / 2}$. In traditional experiments to measure the uniaxial deformation, such as the Texture Analyzer, the measurements are reliable only as measures for the so-called engineering stress, i.e. the stress based on the initial cross-sectional area, where the filament stretching rheometer applied in the work of Bejenariu et $\mathrm{al}^{1}$ can be used to measure the true stress at a given deformation based on the true crosssectional area. This is done by laser measurements of the diameter at the sample length midpoint ${ }^{16}$.

The add-on device to the filament rheometer is shown in figure 6. First the samples are moulded in a steel mould where a holder is placed at each end. The holder contains holes in order to increase the contact area between the silicone and the holder. The samples with holders in both ends can then be mounted in the filament stretch rheometer and the extensional measurements can then be performed in a very controlled way due to the laser measuring the mid-point of the sample. In figure 7 pictures of the elongational experiment to the maximum strain are shown. When the maximum strain is reached, the flow is reversed with a strict control on the diameter.
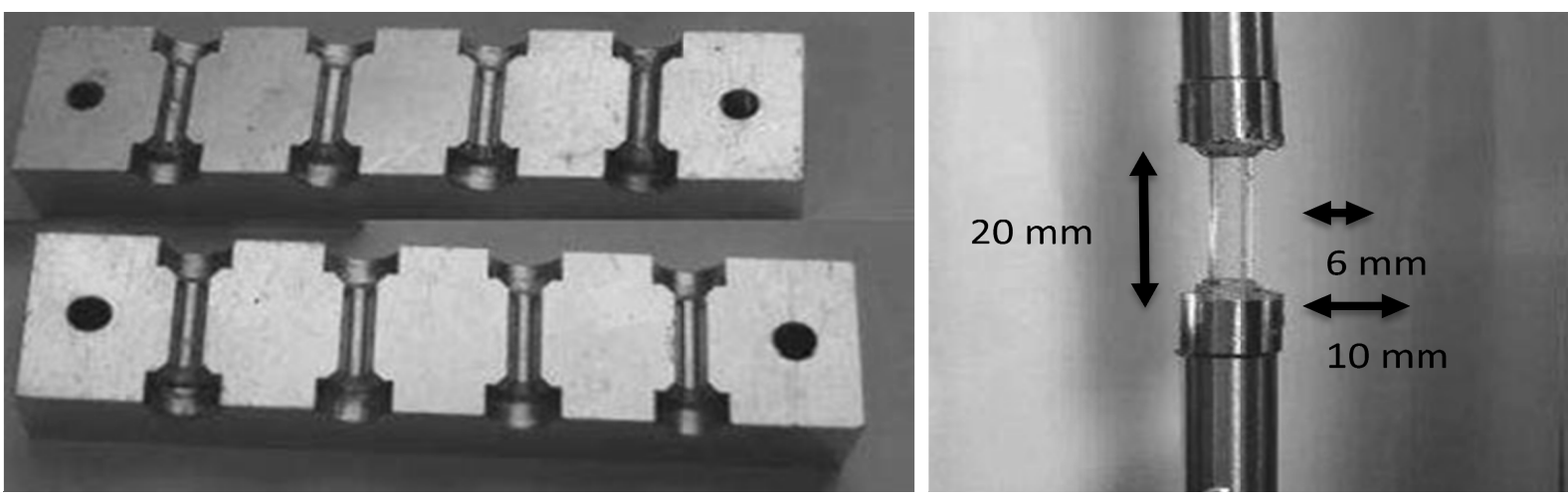

Figure 6: The samples for the LAOE experiments. The mould contains four samples which are reacted in-situ between two sample holders which have holes in both ends. The dimensions are given in the right picture. ${ }^{1}$

In figure 8 the experimental stress-strain curve for a soft silicone elastomer is shown. The elastomer shows strainhardening effects and little tendency to viscous dissipation but the important feature of the data is that the stress is the true stress.

The true stress measurements are very important in order to evaluate the mechanical behavior of the DEAP materials, especially when the flow is a combination of different flows such that the engineering stress measurements have to be translated into different geometries, which is difficult and very prone to large uncertainties. Flows that combine uniaxial and planar elongation extensions are experienced e.g. in the Danfoss Polypower film where the corrugations on the film to a certain degree restricts movement in direction of the corrugation lines on the film but since the film is soft the pattern does not completely suppress the movement in this direction ${ }^{17}$. 
Return to your MySPIE To Do List at http://myspie.org and approve or disapprove this submission. Your manuscript will not be published without this approval. Please contact author_help@spie.org with any questions or concerns.

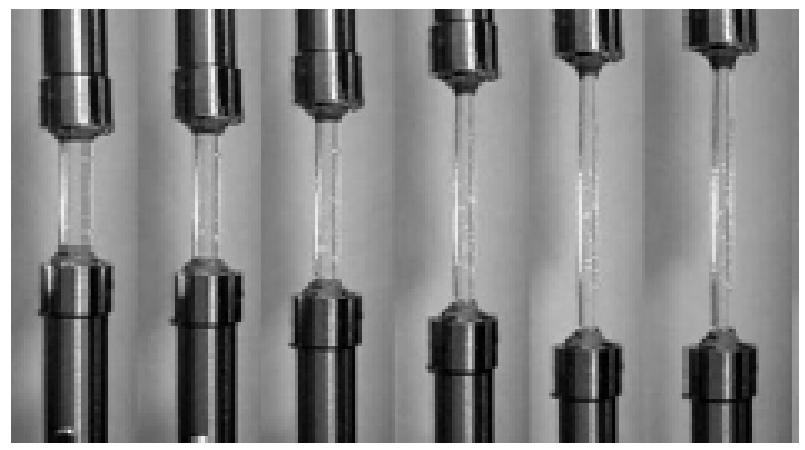

Figure 7. Photos of the dynamics of a soft silicone elastomer (3-functional crosslinker with a stoichiometry of $\mathrm{r}=1.17$ ) subjected to a LAOE experiment. An amplitude of $\lambda=0.7$ and frequency of $v=0.02 \mathrm{~s}^{-1}$ are applied at $23^{\circ} \mathrm{C}$. The time between consecutive pictures is 5 seconds. From the left to the right photo, a half (steady) cycle is shown. ${ }^{1}$

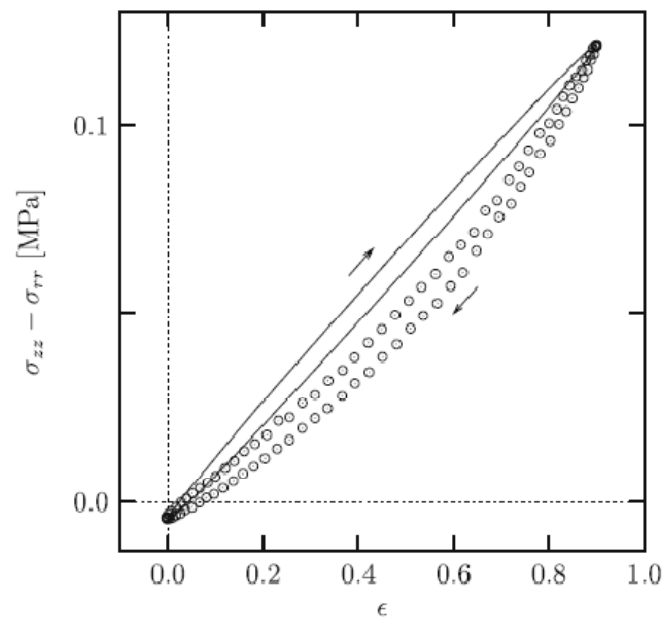

Fig. 8 Lissajous plot (stress strain plot) of the data from silicone elastomers. ${ }^{1}$ The strain at the axis is as in figure 5 the Hencky strain, i.e. the logaritmic strain, such that $\varepsilon=0.9$ means a relative extension in the $z$-direction of $\lambda=\exp (0.9)=2.5$.

\section{CONCLUSION}

LVE data reveals the time-scales within the material as well as the Young's modulus. These informations combined with the data obtained from large amplitude oscillatory uniaxial and planar elongational measurements allows for modeling of the mechanical behavior of the soft elastomers in all operation modes of DEAPs as both methods directly give material functions as well as a measure of the dissipated energy within one cycle of movement. It is also suggested that the methods can be used to test mechanical breakdown in different flow modes of the elastomers. 
Return to your MySPIE To Do List at http://myspie.org and approve or disapprove this submission. Your manuscript will not be published without this approval. Please contact author_help@spie.org with any questions or concerns.

\section{AKNOWLEDGEMENTS}

The author would like to acknowledge the financial support from the Danish National Advanced Technology Foundation.

\section{REFERENCES}

[1] Bejenariu, A.G., Rasmussen, H.K., Skov. A,L., Hassager, O. and Frankaer, S.M., "Large amplitude oscillatory extension of soft polymeric networks," Rheologica Acta (49), 807-14 (2010).

[2] Jensen, M.K., Hassager, O., Rasmussen, H.K., Skov, A.L., Bach, A. and Koldbech, H., "Planar elongation of soft polymeric networks," Rheologica Acta (49), 1-13 (2010).

[3] Jensen, M.K., Rasmussen, H.K., Skov, A.L. and Hassager, O., "Reversed planar elongation of soft polymeric networks," Rheologica Acta (50), 729-40 (2011).

[4] Jensen, M.K., Bach, A., Hassager, O. and Skov, A.L., "Linear rheology of cross-linked polypropylene oxide as a pressure sensitive adhesive," International Journal of Adhesion and Adhesives (29), 687-93 (2009).

[5] Larsen, A.L., Hansen, K., Sommer-Larsen, P., Hassager, O., Bach, A., Ndoni, S., et al., "Elastic properties of nonstoichiometric reacted PDMS networks," Macromolecules (36), 10063-70 (2003).

[6] Wilder, E.A., Guo, S., Lin-Gibson, S., Fasolka, M.J. and Stafford, C.M, "Measuring the modulus of soft polymer networks via a buckling-based metrology," Macromolecules (39), 4138-43 (2006).

[7] Ogden, R.W., "Recent advances in the phenomenological theory of rubber elasticity," Rubber Chemistry and Technology (59), 361-83 (1986).

[8] Chambon, F. and Winter, H.H., "Linear viscoelasticity at the gel point of a cross-linking PDMS with imbalanced stoichiometry," Journal of Rheology (31), 683-97 (1987).

[9] Hansen, R., Skov, A.L. and Hassager, O., "Constitutive equation for polymer networks with phonon fluctuations,". Physical Review E. (77) (2008).

[10] Xing, X., Goldbart, P.M. and Radzihovsky, L., "Thermal fluctuations and rubber elasticity," Physical Review Letters (98) (2007).

[11] Rubinstein, M. and Panyukov, S., "Elasticity of polymer networks," Macromolecules (35), 6670-86(2002).

[12] Larsen, A.L., Sommer-Larsen, P. and Hassager, O., "How to tune rubber elasticity," Smart Structures and Materials Conference. San Diego, 108-17, (2004).

[13] Wang, S.H. and Mark, J.E., "Unimodal and bimodal networks of poly(dimethyl-siloxane) in pure shear," Journal of Polymer Science Part B-Polymer Physics (30), 801-7 (1992).

[14] Xu, P. and Mark, J.E., "Biaxial extension studies using inflation of sheets of unimodal model networks," Rubber Chemistry and Technology (63), 276-84 (1990).

[15] Laun, H.M. and Schuch, H., "Transient elongational viscousities and drawability of polymer melts," Journal of Rheology (33), 119-75(1989).

[16] Bach, A., Rasmussen, H.K. and Hassager, O., "Extensional viscosity for polymer melts measured in the filament stretching rheometer," Journal of Rheology (47), 429-41 (2003).

[17] Benslimane, M., Kiil, H-E. and Tryson, M.J., "Electro-mechanical properties of novel large strain PolyPower film and laminate components for DEAP actuator and sensor applications," Conference on Electroactive Polymer Actuators and Devices (EAPAD), San Diego, CA (2010). 